\title{
Do recombinant-engineered nanoparticle vaccines hold promise for the prevention of respiratory syncytial virus?
}

\author{
"It holds a high promise that respiratory syncytial virus vaccines based \\ on recombinant nanoparticles can be developed into a more effective \\ vaccine than natural infection to prevent respiratory syncytial virus \\ infection."
}

\begin{abstract}
First draft submitted: 19 December 2015; Accepted for publication: 4 January 2016; Published online: 19 February 2016
\end{abstract}

Keywords: recombinant nanoparticles $\bullet$ RSV $\bullet$ vaccines

\section{Safe \& effective RSV vaccines: challenges}

Human respiratory syncytial virus (RSV) can cause severe lung disease such as bronchiolitis and pneumonia in infants, young children, immunocompromised patients and in the elderly. Symptomatic infections are estimated to be 64 million annually worldwide, resulting in approximately 196,000 yearly fatalities [1]. During the 1960s, a formalininactivated RSV vaccine (FI-RSV) with alum adjuvant formulation was used to vaccinate young age children less than 6 months of age, which caused severe respiratory disease requiring hospitalizations and causing two deaths upon natural RSV infection [2]. Since this tragic failure of FI-RSV vaccine trials due to vaccine-enhanced disease (VED) upon infection, there is no licensed vaccine against RSV. In addition to VED, there are several hurdles for developing RSV vaccines. Severe respiratory RSV infection occurs in neonates and young infants. Some children and adults are re-infected every 3-10 years often with severe manifestation of sinus and/or asthma disease complications. Thus, the major challenges for developing RSV vaccines include protection of young infants, inability of natural infection-induced immunity to prevent reinfection and notorious legacy of VED.

\section{Recombinant RSV nanoparticle vaccines in clinical trials}

The RSV fusion (F) glycoprotein mediates viral entry into the target cells, is a target by neutralizing antibodies and conserved in both RSV A and B strains. The monoclonal antibody palivizumab that has neutralizing activity by recognizing a single epitope on RSV F can prevent severe disease in high-risk infants by passive immunoprophylaxis. Purified RSV F protein-based vaccines were formulated with or without alum adjuvant, and administered to humans to assess their efficacies [3]. F protein vaccines were able to induce neutralizing antibodies without displaying adverse events in seropositive children. The best result was shown at a high dose without alum formulation. Approximately afourfold increase in neutralizing antibody titers was observed in $50 \%$ vaccinated individuals. However, the efficacy of $F$ protein vaccines on preventing RSV infection was not sufficiently high enough to justify continued investigation. Soluble RSV protein vaccines were demonstrated to induce pulmonary disease of VED after RSV infection in animal models $[4,5]$.

A precursor (F0) of RSV $\mathrm{F}$ has been synthesized, posttranslationally cleaved at Arg109 and Arg136 by host furin proteases and processed into a shorter F2 polypeptide at the $\mathrm{N}$-terminus linked by two disulfides to a longer F1 fragment with fusion and transmembrane domains. An RSV F nanoparticle vaccine [6] was developed by Novavax, a biotechnology company. First, RSV F was engineered to mutate the cleavage site (Arg136) near to the $\mathrm{F} 1 \mathrm{~N}$-terminus and to delete a part of the F1 hydrophobic fusion domain [6].

\section{Young-Tae Lee}

Institute of Biomedical Sciences, Georgia State University, Atlanta, GA 30303, USA

Young-Man Kwon Institute of Biomedical Sciences, Georgia State University, Atlanta, GA 30303, USA

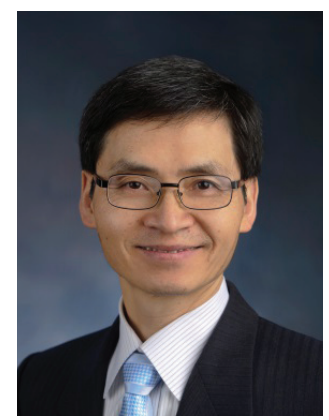

Sang-Moo Kang Author for correspondence: Institute of Biomedical Sciences, Georgia State University, Atlanta, GA 30303, USA

Tel.: +1 4044133588 Fax: +1 4044133580 skang24@gsu.edu 
Recombinant RSV F with near full-length disulfidelinked subunits of F2 and F1 was expressed on the surface of insect cells in a membrane-anchored homotrimer [6]. Insect cell membranes expressing recombinant RSV F were solubilized with nonionic detergent and subjected to a series of chromatography processes to purify RSV F-detergent complex fractions. Recombinant RSV F nanoparticles were formed into a pinlike rosette structure with approximately $40 \mathrm{~nm}$ after removal of the detergent [6].

\section{"Recent progress in developing respiratory syncytial virus vaccines utilizing new advanced technologies is highly promising toward the goal of preventing respiratory syncytial virus disease."}

The Phase I clinical study was successfully carried out, demonstrating the safety and immunogenicity of the RSV F nanoparticle vaccine candidate in healthy adults $18-19$ years of age [7]. An extended clinical study of RSV F nanoparticle vaccine with 350 healthy women (18-35 years) of childbearing age was recently published, demonstrating that the vaccine is safe, immunogenic and can reduce RSV infection [8]. Novavax released the data of a Phase II trial in press, reporting the safety and immunogenicity of RSV F nanoparticle vaccines $(120 \mu \mathrm{g}$ of RSV F with $0.4 \mathrm{mg}$ alum adjuvant) in 50 healthy pregnant women in their third trimester. Maternally transferred RSV specific and neutralizing antibodies with a half-life between 30 and 41 days were observed in infants born to vaccinated mothers with RSV F nanoparticle vaccine. Also, Novavax announced the grant from the Bill \& Melinda Gates Foundation to support the Phase III clinical trial studies of the RSV $F$ vaccine in pregnant women for maternal immunization, expecting the protection of young infants from RSV infection.

\section{Recombinant virus-like nanoparticle vaccines presenting RSV glycoproteins in preclinical studies}

Virus-like nanoparticles (VLPs) can be self-assembled in cells expressing viral structural proteins and present repeating protein antigen arrays similar to those of virus in structure and morphology. The size of VLPs is in a range of approximately $100 \mathrm{~nm}$ depending on the viral core proteins driving the VLP assembly and formation. Newcastle disease virus (NDV) or influenza virus core proteins have been utilized to present RSV F and/or G glycoproteins on the VLP membrane surfaces because of poor capability of RSV structural proteins to form VLPs. For efficient incorporation into NDV-VLPs, chimeric constructs were engineered by fusing the sequences encoding the ectodomains of RSV G or F to the transmembrane and cytoplasmic domains of the NDV glycoproteins [9]. Transfection of avian cells with DNA plasmids encoding NDV structural proteins (NP, M) and RSV chimeric G and/or $\mathrm{F}$ glycoproteins generated VLPs containing the RSV G or both F and G proteins [9,10]. Chimeric RSV-NDV VLPs were shown to be more effective than live RSV in eliciting RSV-neutralizing antibodies and high ratios of $\operatorname{IgG} 2 \mathrm{a} / \mathrm{IgG} 1$ isotypes indicating T-helper type 1 (Th1) immune responses, and inhibiting viral replication in mice upon challenge. VLP-associated VED as assessed by pulmonary histopathological analysis was not observed in comparison with FI-RSV in BALB/c mice. Neutralizing antibodies induced by chimeric RSV-NDV VLPs were lasted for 14 months, whereas those by live RSV declined after 3 months [11]. Also, RSV-NDV VLPs that were engineered to present a prefusion form of RSV $F$ effectively induced neutralizing antibodies after immunization of mice or cotton rats in the absence of adjuvants and confer protection without VED upon RSV challenge $[12,13]$.

Coexpression of RSV F or G and influenza virus M1 matrix protein in insect cells using the baculovirus expression system resulted in efficient release of VLPs presenting RSV F or G, which are effective in inducing Th1-type antibody responses and inhibiting lung viral replication in mice [14]. A combination of RSV $F$ and G VLPs plus F DNA plasmids was effective in inducing RSV-neutralizing antibodies, Th1-type IFN- $\gamma$ secreting CD8 $\mathrm{T}$ cells and CD11 $\mathrm{c}^{+}$phenotypic cells in the airways and preventing eosinophilic pulmonary histopathology after RSV challenge of mice, even better than the immunity by live RSV reinfections [15,16]. Mice that were vaccinated 12 months earlier with RSV F and G VLPs maintained protective immunity in contrast to FI-RSV causing VED characterized by eosinophils, IL-4-producing CD4 $\mathrm{T}$ cells, proinflammatory cytokines, chemokines and plasmacytoid dendritic cells (DCs) [17]. Furthermore, live RSV reinfections induced IFN- $\gamma$ and tumor necrosis factor- $\alpha$ producing CD $4 \mathrm{~T}$ cells and CD11 $\mathrm{b}^{+}$ DCs at high levels, which might be contributing to RSV disease and pulmonary VED despite lung viral clearance after challenge [18]. In contrast, RSV F VLP immunization of mice induced $\mathrm{CD} 8 \alpha^{+} \mathrm{DCs}$ and IFN- $\gamma$ producing CD8 T-cell responses in the lungs, preventing pulmonary histopathology of VED [18]. Thus, F VLP nanoparticle vaccination can induce distinct innate and adaptive cellular subsets preventing pulmonary VED in mice, which are different from FI-RSV vaccination and live RSV reinfections, after RSV challenge. 


\section{Future prospects}

Recent progress in developing RSV vaccines utilizing new advanced technologies is highly promising toward the goal of preventing RSV disease. The Novavax RSV $\mathrm{F}$ nanoparticle vaccine candidate produced in insect cells is advancing to the Phase III clinical trial of maternal immunization in pregnant women, expecting protection against RSV in neonates and young infants. The RSV F nanoparticle vaccine can be safer and more effective in eliciting palivizumab-like RSV-neutralizing antibodies than FI-RSV or live RSV.

There is still a challenge to induce immunity in young infants and children after 3 months of age when maternal antibodies wane below the protection limit. It needs to be investigated whether maternal antibodies or preexisting immunity may have inhibitory effects on the efficacy of active RSV vaccination in young children. Use of the alum adjuvant formulation in the RSV F vaccination may have a safety concern due to its biased induction of Th2-type immune responses associated with pulmonary VED in seronegative children upon RSV infection.

\section{References}

1 Nair H, Nokes DJ, Gessner BD et al. Global burden of acute lower respiratory infections due to respiratory syncytial virus in young children: a systematic review and meta-analysis. Lancet 375(9725), 1545-1555 (2010).

2 Kim HW, Canchola JG, Brandt CD et al. Respiratory syncytial virus disease in infants despite prior administration of antigenic inactivated vaccine. Am. J. Epidemiol. 89(4), 422-434 (1969).

3 Groothuis JR, King SJ, Hogerman DA, Paradiso PR, Simoes EA. Safety and immunogenicity of a purified F protein respiratory syncytial virus (PFP-2) vaccine in seropositive children with bronchopulmonary dysplasia. J. Infect. Dis. 177(2), 467-469 (1998).

4 Murphy BR, Sotnikov AV, Lawrence LA, Banks SM, Prince GA. Enhanced pulmonary histopathology is observed in cotton rats immunized with formalin-inactivated respiratory syncytial virus (RSV) or purified F glycoprotein and challenged with RSV 3-6 months after immunization. Vaccine 8(5), 497-502 (1990).

5 Connors M, Collins PL, Firestone CY et al. Cotton rats previously immunized with a chimeric RSV FG glycoprotein develop enhanced pulmonary pathology when infected with RSV, a phenomenon not encountered following immunization with vaccinia - RSV recombinants or RSV. Vaccine 10(7), 475-484 (1992).

6 Smith G, Raghunandan R, Wu Y et al. Respiratory syncytial virus fusion glycoprotein expressed in insect cells form protein nanoparticles that induce protective immunity in cotton rats. PLoS ONE 7(11), e50852 (2012).

7 Glenn GM, Smith G, Fries L et al. Safety and immunogenicity of a Sf9 insect cell-derived respiratory syncytial virus fusion protein nanoparticle vaccine. Vaccine 31(3), 524-532 (2013).
Recombinant-engineered RSV VLP vaccines do not require adjuvants for eliciting antibodies to a level of protection. Also, VLPs presenting a stabilized prefusion form of RSV F appear to be quite promising candidates. Manufacturing and regulatory processes should be followed to advance VLP-based RSV vaccines for clinical trials. It holds a high promise that RSV vaccines based on recombinant nanoparticles can be developed into a more effective vaccine than natural infection to prevent RSV infection.

\section{Financial \& competing interests disclosure}

This work was supported by NIH/NIAID grants Al105170 (SM Kang), Al119366 (SM Kang), and Al093772 (SM Kang). The authors have no other relevant affiliations or financial involvement with any organization or entity with a financial interest in or financial conflict with the subject matter or materials discussed in the manuscript apart from those disclosed.

No writing assistance was utilized in the production of this manuscript.

8 Glenn GM, Fries LF, Thomas DN et al. A randomized, blinded, controlled, dose-ranging study of a respiratory syncytial virus recombinant fusion (F) nanoparticle vaccine in healthy women of childbearing age. J. Infect. Dis. 213(3), 411-422 (2016).

9 Mcginnes LW, Gravel KA, Finberg RW et al. Assembly and immunological properties of Newcastle disease virus-like particles containing the respiratory syncytial virus $F$ and $G$ proteins. J. Virol. 85(1), 366-377 (2011).

10 Murawski MR, Mcginnes LW, Finberg RW et al. Newcastle disease virus-like particles containing respiratory syncytial virus $\mathrm{G}$ protein induced protection in BALB/c mice, with no evidence of immunopathology. J. Virol. 84(2), 1110-1123 (2010).

11 Schmidt MR, Mcginnes LW, Kenward SA, Willems KN, Woodland RT, Morrison TG. Long-term and memory immune responses in mice against Newcastle disease virus-like particles containing respiratory syncytial virus glycoprotein ectodomains. J. Virol. 86(21), 11654-11662 (2012).

12 Mcginnes Cullen L, Schmidt MR, Kenward SA, Woodland RT, Morrison TG. Murine immune responses to viruslike particle-associated pre- and postfusion forms of the respiratory syncytial virus F protein. J. Virol. 89(13), 6835-6847 (2015).

13 Cullen LM, Blanco JC, Morrison TG. Cotton rat immune responses to virus-like particles containing the pre-fusion form of respiratory syncytial virus fusion protein. J. Transl. Med. 13(1), 350 (2015).

14 Quan FS, Kim Y, Lee $S$ et al. Viruslike particle vaccine induces protection against respiratory syncytial virus infection in mice. J. Infect. Dis. 204(7), 987-995 (2011).

15 Hwang HS, Kwon YM, Lee JS et al. Co-immunization with virus-like particle and DNA vaccines induces protection 
against respiratory syncytial virus infection and bronchiolitis. Antiviral Res. 110C, 115-123 (2014).

16 Ko EJ, Kwon YM, Lee JS et al. Virus-like nanoparticle and DNA vaccination confers protection against respiratory syncytial virus by modulating innate and adaptive immune cells. Nanomedicine 11(1), 99-108 (2015).

17 Lee YT, Ko EJ, Hwang HS et al. Respiratory syncytial viruslike nanoparticle vaccination induces long-term protection without pulmonary disease by modulating cytokines and T-cells partially through alveolar macrophages. Int. J. Nanomedicine 10, 4491-4505 (2015).

$18 \mathrm{Kim} \mathrm{KH}$, Lee YT, Hwang HS et al. Virus-like particle vaccine containing the $\mathrm{F}$ protein of respiratory syncytial virus confers protection without pulmonary disease by modulating specific subsets of dendritic cells and effector $T$ cells. J. Virol. 89(22), 11692-11705 (2015). 